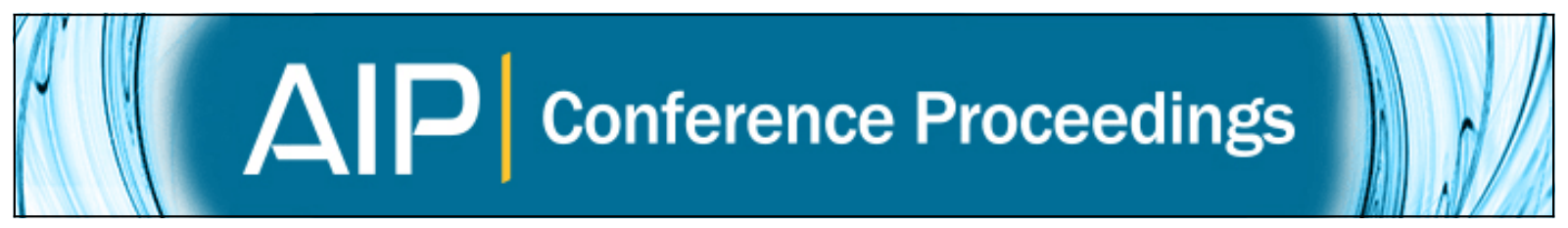

Inducing pivot variables and non-centrality parameters in elliptical distributions

Dário Ferreira, Sandra S. Ferreira, Célia Nunes, and Sónia Inácio

Citation: AIP Conference Proceedings 1558, 833 (2013); doi: 10.1063/1.4825625

View online: http://dx.doi.org/10.1063/1.4825625

View Table of Contents: http://scitation.aip.org/content/aip/proceeding/aipcp/1558?ver=pdfcov

Published by the AIP Publishing

Articles you may be interested in

Analytical solutions of a generalized non-central potential in $\mathrm{N}$-dimensions

J. Math. Phys. 55, 102105 (2014); 10.1063/1.4899084

Cross-over between central and non-central conservative effective forces in a modulated colloidal fluid

J. Chem. Phys. 139, 204903 (2013); 10.1063/1.4832735

Vapor nucleation on a wettable nanoparticle carrying a non-central discrete electric charge

J. Chem. Phys. 138, 194708 (2013); 10.1063/1.4804655

Confidence regions for variance components using inducing pivot variables

AIP Conf. Proc. 1479, 1682 (2012); 10.1063/1.4756493

Inference with Inducer Pivot Variables, an Application to the One-Way ANOVA

AIP Conf. Proc. 1389, 1631 (2011); 10.1063/1.3636921 


\title{
Inducing Pivot Variables and Non-centrality Parameters in Elliptical Distributions
}

\author{
Dário Ferreira*, Sandra S. Ferreira*, Célia Nunes* and Sónia Inácio ${ }^{\dagger}$ \\ * Department of Mathematics and Center of Mathematics, University of Beira Interior, Covilhã, Portugal \\ ${ }^{\dagger}$ Department of Mathematics and CMA, Faculty of Science and Technology, New University of Lisbon, Portugal
}

\begin{abstract}
We used inducing pivot variables to derive confidence intervals for the non-centrality parameters of samples with elliptical errors. A numerical application is presented.

Keywords: Pivot variables, inference, variance components AMS: $62 \mathrm{k} 15,62 \mathrm{E} 15,62 \mathrm{H} 10,62 \mathrm{H} 15,62 \mathrm{~J} 10$
\end{abstract}

\section{INTRODUCTION}

Inducing pivot variables are functions of statistics and parameters with known distributions which induce probability measures. As it may be seen in [2], these variables may be used to carry out inference. to derive confidence intervals for the non centrality parameters of samples with elliptical errors.

The remainder of this article is arranged as follows. In the next section we present results on spherical and elliptical distributions. Next we introduce the concept of inducing pivot variable and show how to construct confidence intervals and test hypothesis for non-centrality parameters of samples with elliptical errors. Finally we present a numerical application.

\section{SPHERICAL AND ELLIPTICAL DISTRIBUTIONS}

Let $\boldsymbol{X}$ be a $k \times 1$ random vector and its support be the set of $k$-dimensional real vectors

$$
R(\boldsymbol{X})=\mathbb{R}^{k}
$$

Consider $\boldsymbol{\mu}$ a $k \times 1$ vector and $\boldsymbol{V}$ a $k \times k$ symmetric and positive definite matrix, that is,

$$
\boldsymbol{V}=\boldsymbol{V}^{\prime}
$$

where $\boldsymbol{V}^{\prime}$ denotes the transpose of matrix $\boldsymbol{V}$, and $\boldsymbol{S}^{\prime} \boldsymbol{V} \boldsymbol{S}$ is positive for any non-zero column vector $\boldsymbol{S}$ of $k$ real numbers. Vector $\boldsymbol{X}$ has a multivariate normal distribution with mean vector $\boldsymbol{\mu}$ and covariance matrix $\boldsymbol{V}$ if its joint density function is

$$
f(\boldsymbol{X})=(2 \pi)^{-\frac{K}{2}}|\operatorname{det}(\boldsymbol{V})|^{-\frac{1}{2}} \exp \left(-\frac{1}{2}(\boldsymbol{X}-\boldsymbol{\mu})^{\prime} \boldsymbol{V}^{-1}(\boldsymbol{X}-\boldsymbol{\mu})\right) .
$$

We indicate that $\boldsymbol{X}$ has a multivariate normal distribution with mean vector $\boldsymbol{\mu}$ and covariance matrix $\boldsymbol{V}$ by

$$
\boldsymbol{X} \sim N(\boldsymbol{\mu}, \boldsymbol{V})
$$

The $k$ random variables $X_{1}, \ldots, X_{k}$ constituting the vector $\boldsymbol{X}$ are said to be jointly normal.

The class of spherical distributions is an extension of the class of multivariate normal distributions. Vector $\boldsymbol{X}$ is said to have a spherical distribution if $\boldsymbol{X}$ and $\boldsymbol{Q X}$ have the same distribution for all orthogonal $k \times k$ matrices $\boldsymbol{Q}$, see [4]. If $\boldsymbol{X}$ is a continuous random vector with a spherical distribution, then due to the equality

$$
Q^{\prime} \boldsymbol{Q}=\boldsymbol{I},
$$


its density function depends on the argument $\boldsymbol{X}$ through the value of $\boldsymbol{X}^{\prime} \boldsymbol{X}$ and is of the form $g\left(\boldsymbol{X}^{\prime} \boldsymbol{X}\right)$ for some nonnegative function $g(\cdot)$. A study about the density of a spherical distribution may be seen, for example in [1]. If two random vectors $\boldsymbol{X}$ and $\boldsymbol{Y}$ have the same distribution we use the notation $\boldsymbol{X} \stackrel{d}{=}$.

Let the k-vector $\boldsymbol{X}$ be spherically distributed. Then $\boldsymbol{X}$ has the stochastic representation

$$
\boldsymbol{X} \stackrel{d}{=} \boldsymbol{R},
$$

where $\boldsymbol{U}$ is uniformly distributed on the unit sphere, $\boldsymbol{R} \sim F(\boldsymbol{X})$ is independent of $\boldsymbol{U}$, and $F(\boldsymbol{X})$ is a distribution function

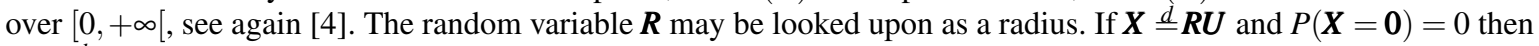
$\|\boldsymbol{X}\| \stackrel{d}{=} \boldsymbol{R}$, where $\|\cdot\|$ denotes the usual Euclidian norm.

Vector $\boldsymbol{X}$ is said to have an elliptical distribution, with mean vector $\boldsymbol{\mu}$ and covariance matrix $\boldsymbol{V}$, if $\boldsymbol{X}$ has the same distribution as $\boldsymbol{\mu}+\boldsymbol{A} \boldsymbol{Y}$, where $\boldsymbol{Y}$ has a spherical distribution and $\boldsymbol{A}$ is a $k \times p$ matrix, with

$$
\boldsymbol{A A ^ { \prime }}=\boldsymbol{V}
$$

and $\operatorname{rank}(\boldsymbol{V})=p$.

The multivariate normal distribution belongs to the class of elliptical distributions since, if $\boldsymbol{X} \sim N(\boldsymbol{\mu}, \boldsymbol{V})$, the vector $\boldsymbol{X}$ can be represented as $\boldsymbol{X}=\boldsymbol{\mu}+\boldsymbol{A} \boldsymbol{Y}$, where $\boldsymbol{Y} \sim N(\mathbf{0}, \boldsymbol{I})$ and $\boldsymbol{A} \boldsymbol{A}^{\prime}=\boldsymbol{V}$.

\section{INDUCING PIVOT VARIABLES}

As already stated in the introduction, pivot variables are functions of statistics and parameters with known distributions. For example, if $S$ is distributed as the product by $\gamma$ of a central chi-square with $g$ degrees of freedom, $S \sim \gamma \chi_{g}^{2}$, then

$$
Z=\frac{S}{\gamma}
$$

is distributed as a central chi-square with $g$ degrees of freedom, being therefore a pivot variable. Now, let $\mathscr{B}_{r}$ be the $\sigma$-algebra of the borelian sets in $\mathbb{R}^{r}$, see [7], and the parameter space $\Theta \in \mathscr{B}_{r}$. According to [3] the pivot variable

$$
\boldsymbol{Z}=g(\boldsymbol{Y}, \boldsymbol{\theta})
$$

is an inducing pivot variable if, for any realization $\boldsymbol{y}$ of $\boldsymbol{Y}$ the function

$$
l(\boldsymbol{\theta} \mid \boldsymbol{y})=g(\boldsymbol{y}, \boldsymbol{\theta})
$$

has an inverse measurable function $h(\boldsymbol{z} \mid \boldsymbol{y})$ in $\mathscr{B}_{r}$.

In the next section we will use inducing pivot variables to derive confidence intervals and test hypothesis for noncentrality parameters when we are dealing with elliptical distributions.

\section{INFERENCE FOR NON-CENTRALITY PARAMETERS}

Consider the random vector

$$
\boldsymbol{X} \sim N(\boldsymbol{\mu}, \theta \boldsymbol{I})
$$

independent of $S$, which is distributed as the product by $\theta$ of a central chi-square with $g$ degrees of freedom,

$$
S \sim \theta \chi_{g}^{2}
$$

If we consider

$$
W=\frac{\|\boldsymbol{X}\|^{2}-\|\boldsymbol{\mu}\|^{2}}{2\|\boldsymbol{\mu}\|},
$$

with $\|\boldsymbol{\mu}\| \rightarrow \infty$, then $W$ will be approximately normally distributed, with mean 0 and variance $\theta, W \stackrel{\circ}{\sim} N(0, \theta)$, and independent of $S$, see [5] and [6]. Therefore $\mathscr{F}=g \frac{W^{2}}{S}$ will have $F$ distribution with 1 and $g$ degrees of freedom and non-centrality parameter $\delta, \mathscr{F} \sim F(. \mid 1, g, \delta)$, where

$$
\delta=\frac{\|\boldsymbol{\mu}\|^{2}}{\theta} .
$$


Moreover, if

$$
T=\sqrt{g} \frac{W}{\sqrt{S}},
$$

$T$ will have $t$-distribution, with $g$ degrees of freedom, $T \sim t_{g}$.

Now, if $\boldsymbol{x}, s, t$ and $w$ are realizations of $\boldsymbol{X}, S, T$ and $W$ respectively, from (13) and (15) we will have

$$
\frac{\|\boldsymbol{x}\|^{2}-\|\boldsymbol{\mu}\|^{2}}{2\|\boldsymbol{\mu}\| \sqrt{s}}=\frac{t}{\sqrt{g}},
$$

which is equivalent to having

$$
\|\boldsymbol{\mu}\|^{2}+\frac{2 t \sqrt{s}\|\boldsymbol{\mu}\|}{\sqrt{g}}-\|\boldsymbol{x}\|^{2}=0 .
$$

Solving (17) in order to $\|\boldsymbol{\mu}\|$, we get

$$
\|\boldsymbol{\mu}\|=\frac{-t \sqrt{s}}{\sqrt{g}}+\sqrt{\frac{t^{2} s}{g}+\|\boldsymbol{x}\|^{2}}=-w+\sqrt{w^{2}+\|\boldsymbol{x}\|^{2}},
$$

so

$$
\|\boldsymbol{\mu}\|^{2}=\left(-w+\sqrt{w^{2}+\|\boldsymbol{x}\|^{2}}\right)^{2} .
$$

If we consider the pivot variable $Z=\frac{S}{\theta} \sim \chi_{g}^{2}$ and $W \sim N(0, \theta)$, we may induce probability measures for

$$
\delta=\frac{\|\boldsymbol{\mu}\|^{2}}{\theta}=\frac{\left(-w+\sqrt{w^{2}+\|\boldsymbol{x}\|^{2}}\right)^{2}}{\frac{s}{Z}},
$$

using large samples $D=\left\{D_{1}, \ldots, D_{n}\right\}$, with

$$
D_{u}=\frac{\left(-W_{u}+\sqrt{W_{u}^{2}+\|\boldsymbol{x}\|^{2}}\right)^{2}}{\frac{s}{Z_{u}}}, u=1, \ldots, n,
$$

where $Z_{u} \sim \chi_{g}^{2}, W_{u} \sim N\left(0, \theta_{u}\right)$ and $\theta_{u}=\frac{s}{Z_{u}}, u=1, \ldots, n$.

Let $F(x)$ be the distribution of $D, x_{p}$ the quantile for probability $p, F_{n}$ be the empirical distribution of $D$ and $x_{n, p}$ the $F_{n}$ quantile for probability $p$. Now, using the reverse Glivenko-Cantelli theorem, see [2], we may estimate the quantiles $x_{p}$ of $F_{x}$ from the quantiles $x_{n, p}$ of $F_{n}$. Therefore, we may construct confidence intervals $\left[x_{n, \frac{\alpha}{2}} ; x_{n, 1-\frac{\alpha}{2}}\right] ;\left[0 ; x_{n, 1-\alpha}\right]$ and $\left[x_{n, \alpha} ;+\infty[\right.$ for $\delta$, with (estimated) confidence level $1-\alpha$. These confidence intervals allow us to test hypothesis

$$
H_{0}: \delta=\delta_{0}
$$

against

$$
H_{1, j}: \delta \neq \delta_{0} ; \delta>\delta_{0} \text { and } \delta<\delta_{0} .
$$

We reject the test hypothesis if $\delta_{0}$ is not contained in the corresponding confidence interval. Thus, by duality, we obtain tests with (approximate) confidence level $\alpha$.

\section{NUMERICAL APPLICATION}

In order to obtain confidence intervals for $\delta$, defined in (14), when the random vector $\boldsymbol{X}$, in (11), and the sum of squares for the error, $S$, in (12), are known, we used the R software to generate $Z_{u} \sim \chi_{g}^{2}$ and $\boldsymbol{X}_{u}$, with components $X_{u, 1}, \ldots, X_{u, 10}$, considering

$$
X_{u, 1}, \ldots, X_{u, 9} \sim N\left(0, \theta_{u}\right) \text { and } X_{u, 10} \sim N\left(\sqrt{\theta_{u}}, \theta_{u}\right),
$$

where $\theta_{u}=\frac{s}{Z_{u}}$ and $u=1, \ldots, 10000$. Note that we considered $\delta=1$ since $\|\boldsymbol{\mu}\|^{2}=\theta$. 
Next we generated $W_{u} \sim N\left(0, \theta_{u}\right), u=1, \ldots, 10000$, and obtained the sample $D=\left\{D_{1}, \ldots, D_{10000}\right\}$, with

$$
D_{u}=\frac{\left(-W_{u}+\sqrt{W_{u}^{2}+\|\boldsymbol{x}\|^{2}}\right)^{2}}{\frac{s}{Z_{u}}}, u=1, \ldots, 10000 .
$$

The obtained values for $x_{n, 0.025}$ and $x_{n, 0.975}$ are presented in Table 1 and Table 2, respectively. These tables also show the considered values for $S$ and for $g$.

TABLE 1. Obtained values for $x_{n, 0.025}$.

\begin{tabular}{crrrrrrrrrrrrr}
\hline & & & \multicolumn{1}{c}{$S$} & \multicolumn{1}{c}{$S$} & & & & & & \\
$g$ & $\mathbf{0 . 5}$ & $\mathbf{1}$ & $\mathbf{2}$ & $\mathbf{4}$ & $\mathbf{8}$ & $\mathbf{1 6}$ & $\mathbf{3 2}$ & $\mathbf{6 4}$ & $\mathbf{1 2 8}$ & $\mathbf{2 5 6}$ & $\mathbf{5 1 2}$ & $\mathbf{1 0 2 4}$ \\
\hline 1 & 0.8301 & 0.8289 & 0.8377 & 0.8348 & 0.8257 & 0.8368 & 0.8290 & 0.8311 & 0.8384 & 0.8350 & 0.8378 & 0.8294 \\
2 & 0.8373 & 0.8381 & 0.8325 & 0.8385 & 0.8362 & 0.8398 & 0.8387 & 0.8539 & 0.8418 & 0.8399 & 0.8387 & 0.8427 \\
4 & 0.8414 & 0.8340 & 0.8385 & 0.8308 & 0.8312 & 0.8310 & 0.8444 & 0.8344 & 0.8303 & 0.8367 & 0.8374 & 0.8251 \\
8 & 0.8367 & 0.8389 & 0.8496 & 0.8301 & 0.8354 & 0.8405 & 0.8252 & 0.8274 & 0.8364 & 0.8363 & 0.8368 & 0.8232 \\
16 & 0.8323 & 0.8331 & 0.8200 & 0.8271 & 0.8334 & 0.8378 & 0.8453 & 0.8353 & 0.8457 & 0.8285 & 0.8321 & 0.8205 \\
32 & 0.8340 & 0.8310 & 0.8399 & 0.8280 & 0.8411 & 0.8232 & 0.8272 & 0.8368 & 0.8435 & 0.8349 & 0.8421 & 0.8334 \\
64 & 0.8313 & 0.8339 & 0.8447 & 0.8421 & 0.8301 & 0.8461 & 0.8338 & 0.8373 & 0.8219 & 0.8434 & 0.8302 & 0.8263 \\
128 & 0.8350 & 0.8408 & 0.8409 & 0.8412 & 0.8342 & 0.8341 & 0.8339 & 0.8372 & 0.8349 & 0.8313 & 0.8279 & 0.8376 \\
\hline
\end{tabular}

TABLE 2. Obtained values for $x_{n, 0.975}$.

\begin{tabular}{|c|c|c|c|c|c|c|c|c|c|c|c|c|}
\hline \multicolumn{13}{|c|}{$S$} \\
\hline$g$ & 0.5 & 1 & 2 & 4 & 8 & 16 & 32 & 64 & 128 & 256 & 512 & 1024 \\
\hline 1 & 1.1923 & 1.1796 & 1.1856 & 1.1927 & 1.1933 & 1.1848 & 1.1872 & 1.1903 & 1.1892 & 1.1971 & 1.1927 & 1.1812 \\
\hline 2 & 1.1854 & 1.1845 & 1.1841 & 1.1826 & 1.1970 & 1.1821 & 1.1854 & 1.1884 & 1.1687 & 1.1765 & 1.1832 & 1.1758 \\
\hline 4 & 1.1898 & 1.1895 & 1.1793 & 1.1665 & 1.1955 & 1.1748 & 1.1906 & 1.1815 & 1.1955 & 1.1723 & 1.1732 & 1.1932 \\
\hline 8 & 1.1841 & 1.1824 & 1.1796 & 1.1862 & 1.1863 & 1.1825 & 1.1881 & 1.1694 & 1.2004 & 1.1834 & 1.1811 & 1.1786 \\
\hline 16 & 1.2057 & 1.1712 & 1.1827 & 1.1990 & 1.1922 & 1.1839 & 1.1814 & 1.1886 & 1.1905 & 1.1846 & 1.1865 & 1.1850 \\
\hline 32 & 1.1992 & 1.1846 & 1.1892 & 1.1801 & 1.1831 & 1.1900 & 1.1796 & 1.1982 & 1.1854 & 1.1912 & 1.1892 & 1.2043 \\
\hline 64 & 1.1784 & 1.1795 & 1.1709 & 1.1850 & 1.1784 & 1.1867 & 1.1734 & 1.1852 & 1.1889 & 1.1896 & 1.1873 & 1.2018 \\
\hline 128 & 1.1935 & 1.1877 & 1.1905 & 1.1927 & 1.1821 & 1.1751 & 1.1697 & 1.1993 & 1.1891 & 1.1745 & 1.1788 & 1.1836 \\
\hline
\end{tabular}

These tables point to the stability of the quantiles we chosen. So, the presented method of constructing confidence intervals, for non-centrality parameters, is usefull and apply when we have elliptical density for the error.

\section{ACKNOWLEDGMENTS}

This work was partially supported by the Center of Mathematics, University of Beira Interior through the project PEst-OE/MAT/UI0212/2011 and by CMA, Faculty of Science and Technology, New University of Lisbon, through the project PEst- OE/MAT/UI0297/2011.

\section{REFERENCES}

1. Fang, K.-T. and Zhang, Y.-T. Generalized Multivariate Analysis. Science Press, Beijing, Springer-Verlag, Berlin 1990.

2. Ferreira, D, Ferreira, Sandra, S, Nunes, C, Fonseca, M. and Mexia, J. T. (2013). Chisquared and Related Inducing Pivot Variables: An Application to Orthogonal Mixed Models. Communications in Statistics - Theory and Methods. To appear

3. Fonseca, M., Mexia, J. T. and Zmyślony, R. (2007). Jordan algebras, generating pivot variables and orthogonal normal models. Journal of Interdisciplinary Mathematics, 1, 305-326.

4. Kollo, T. and von Rosen, D. Advanced Multivariate Statistics with Matrices. Springer-Verlag, Berlin 2005.

5. Mexia J.T. and Oliveira M.M. (2010). Asymptotic linearity and limit distributions, approximations. J. Stat. Plan. Infer. 140 (2), 353-357.

6. Nunes C., Oliveira M.M. and Mexia J.T. (2013). Application domains for the Delta method. Statistics. 47(2), 317-328.

7. Williams, D. Probability with Martingales. Cambridge University Press 1991. 